\title{
Anvil or Onion? Determinism as a Layered Concept
}

\author{
Robert C. Bishop \\ Center for Junior Research Fellows, Box M682 \\ University of Konstanz \\ D-78457 Konstanz, Germany \\ and \\ Faculty of Philosophy \\ University of Oxford \\ 10 Merton Street \\ Oxford OX1 4JJ, United Kingdom
}

\begin{abstract}
Stephen Kellert (1993) has argued that Laplacean determinism in classical physics is actually a layered concept, where various properties or layers composing this form of determinism can be peeled away. Here, I argue that a layered conception of determinism is inappropriate and that we should think in terms of different deterministic models applicable to different kinds of systems. The upshot of this analysis is that the notion of state is more closely tied to the kind of system being investigated than is usually considered in discussions of determinism. So when investigating determinism corresponding changes to the appropriate notion of state-and, perhaps, the state space itself-also need to be considered.
\end{abstract}

\section{Introduction}

Determinism is generally taken to be a metaphysical doctrine about our world. If metaphysical determinism is true, then it is reasonable to think that scientific determinism-the determinism studied in physical theories-would also be true in some form. However, it is a further question as to what properties a theory or model must have in order to be deterministic.

This latter question usually is much easier to judge in the case of concrete theories than the status of scientific or metaphysical determinism in general. Laplace's famous characterization of determinism (1814/1951, p. 4) is an example of scientific determinism based on the paradigm theory of classical particle mechanics (CPM). Mark Stone gave a particularly clear characterization of Laplacean determinism in CPM, where its key properties are seen to form jointly necessary and sufficient conditions for determinism (1989). In contrast, Stephen Kellert more recently analyzed the properties Stone identifies as representing layers or levels of determinism (1993, chapter 2).

In this essay, I examine these two analyses of the properties defining Laplacean determinism, connecting these properties more closely with the mathematical machinery typically employed in physical theories. There are some subtleties regarding the connection between these defining properties and states of systems that call the layered approach into question as a way of conceiving Laplacean determinism, and uncovering these subtleties helps us get clearer about determinism in physical theories. 


\section{Preliminaries}

Theoretical models in physics include equations of motion (often referred to as dynamical or evolution equations) describing the change in time of the relevant variables characterizing the system in question, a complete specification of the initial state referred to as the initial conditions (ICs) for the model and/or a characterization of the boundaries for the model known as the boundary conditions (BCs). A state is taken to be a description of the values of the variables characterizing the system at some time t. As a simple example of a classical model, suppose we wanted to study the firing of a rubber ball at a wall by a cannon. The $\mathrm{BC}$ might be that the wall absorbs no kinetic energy (energy of motion) so that the ball is reflected off the wall with no loss of energy. The ICs would be the initial position and velocity of the ball as it left the mouth of the cannon. The equation of motion would then describe the path of the ball.

It is useful to introduce a distinction that is immediately relevant to physical descriptions, namely the ontic/epistemic distinction. This distinction is applied to states and properties of a physical system. Roughly, ontic states and properties refer to features of physical systems as they are "when nobody is looking," while epistemic states and properties refer to features of physical systems accessible to empirical observation. ${ }^{1}$ Erhard Scheibe (1964/1973) first introduced this distinction and it has been subsequently developed in various versions (Primas 1990 and 1994; Atmanspacher 1994; d'Espagnat 1994). An important special case of ontic states and properties are those that are deterministic and describable in terms of points in an appropriate state space; whereas an important special case of epistemic states and properties are those that are describable in terms of probability distributions (or density operators) on some appropriate state space.

Much of the analysis of physical systems takes place in what is called state space, an abstract mathematical space composed of the variables required to fully specify the state of a system. Each point in this space then represents a possible state of the system at a particular time $\mathrm{t}$ through the values these variables take on at t. When the state of the system is fully characterized by position and momentum variables, the resulting space is often called phase space. In typical dynamical models, the coordinates of such a space are the generalized momenta and positions characterizing the possible states. ${ }^{2}$ A model can be studied in state space by following its trajectory from the initial state $\left(q_{o}, p_{o}\right)$ to some final state $\left(q_{f}, \underline{p}_{f}\right)$. The evolution equations govern the path-the history of state transitions-of the system in state space.

However, note that there are important assumptions being made here. Namely, that a state of a system is characterized by the values of the crucial variables and that a physical state corresponds to a point in state space through these values. This cluster of assumptions can be called the faithful model assumption. This assumption allows us to develop mathematical models for the evolution of these points in state space and such models are taken to represent (perhaps via an isomorphism or through a more complicated relation) the physical systems of interest. In other words, we assume that our mathematical models are faithful representations of physical systems and the state space is a faithful representation of the physical space of the system in question. Hence, we

\footnotetext{
${ }^{1}$ This is not to say that ontic states have no empirical implications. For example, if there are restrictions on the evolution of ontic states, then these limitations may very well be reflected in the observable evolutions of epistemic states.

${ }^{2}$ These generalized coordinates allow for systems to be characterized by variables other than linear momentum and position (e.g., angles and angular momentum).
} 
have our connection between deterministic physical systems and their deterministic models, provided the latter are faithful and have properties rendering them deterministic.

One advantage of working in state space is that it often allows us to study the geometric properties of the trajectories of the system in question without knowing the exact solutions to the dynamical equations. But different kinds of state spaces are of different kinds of usefulness. So one encounters various kinds of spaces such as phase space (momentum vs. position), configuration space (position vs. time), "Liouville" or density space (e.g., particle density vs. position) as well as various kinds of transformed spaces, where, for example, the momentum has been Fourier transformed into a vector characterizing frequencies in the system.

\section{Laplacean Determinism}

Clocks, cannon balls fired from cannons and the solar system are taken to be paradigm examples of deterministic systems in classical physics. In the practice of physics, we are able to give a very general and precise description of deterministic systems conceived of ontically. For definiteness I will focus on classical particle mechanics (CPM), the inspiration for Laplace's famous description. Suppose that the physical state of a system is characterized by the values of the positions and momenta of all the particles composing the system at some time t. Furthermore, suppose that a physical state corresponds to a point in state space in an ontic description through these values (invoking the faithful model assumption). We can then develop deterministic mathematical models for the evolution of these points in state space and three properties have been identified by Stone as playing a crucial role in such descriptions expressing Laplace's vision of determinism (Stone 1989; Kellert 1993; Bishop 2002; Bishop 2003):

(DD) Differential Dynamics: An algorithm relates a state of a system at any given time to a state at any other time and the algorithm is not probabilistic. ${ }^{3}$

(UE) Unique Evolution: A given state is always followed (preceded) by the same history of state transitions. ${ }^{4}$

(VD) Value Determinateness: Any state can be described with arbitrarily small (nonzero) error. ${ }^{5}$

Differential dynamics is motivated by actual physical theories expressed in terms of mathematical equations. These equations, along with ICs and BCs, are required to be nonprobabilistic. This requirement expresses the Laplacean belief that there are no indeterministic elements in CPM like those thought to be present in some versions of quantum mechanics. Such equations describe the individual trajectories of states in state space.

\footnotetext{
${ }^{3}$ Though the term "differential dynamics" might lead one to think only differential equations are in view in this condition, it is actually much more general, allowing difference, integral and integro-differential equations among other possibilities arising in physical theories.

${ }^{4}$ As formulated, UE expresses bidirectional state transitions (future and past). It can easily be recast to allow for unidirectional state transitions (future only or past only).

${ }^{5}$ These descriptions can be ontic or epistemic.
} 
Unique evolution is closely associated with DD and expresses the Laplacean belief that systems in CPM will repeat their behaviors exactly if the same initial and boundary conditions are specified. For example the equations of motion for a frictionless pendulum will produce the same solution for the motion as long as the same initial velocity and initial position are chosen. Roughly the idea is that every time we return the mathematical model to the same initial state (or any state in the history of state transitions), it will undergo the same history of transitions from state to state and likewise for the target system. In other words the evolution will be unique with respect to a particular specification of ICs and BCs. ${ }^{6}$

The importance of UE for determinism will be one of the issues discussed in section 4 , so it is worthwhile to say a bit more about it here. Although a strong requirement, UE is important if physical determinism is to be a meaningful concept. Imagine a typical physical system $\underline{m}$ as a film. Unique evolution means that if we were to start the film over and over at the same frame (returning the system to the same initial state), then $\underline{m}$ would repeat every detail of its total history over and over again and identical copies of the film would produce the same sequence of pictures. So no matter whether we always start Jurassic Park at the beginning frame, the middle frame or any other frame, it plays the same. The T-rex as antihero always saves the day. No new frames are added to the movie nor is the sequence of the frames changed simply by starting it at an arbitrary frame.

By way of contrast, suppose it was the case that returning $\underline{m}$ to the same initial state produced a different sequence of state transitions on some of the runs. Consider a system $\underline{m}$ to be like a device that generates a different sequence of pictures on some occasions when starting from the same initial picture. Imagine further that such a system has the property that simply by choosing to start with any picture normally appearing in the sequence, it is sometimes the case that the chosen picture is not followed by the usual sequence of pictures or that some pictures often do not appear in the sequence or that new ones are added from time to time. Such a system would fail to have UE and would not qualify as a deterministic.

Value determinateness is motivated by the Laplacean belief that there is nothing in principle in CPM preventing mathematical descriptions of arbitrary accuracy. For example the models of CPM all presuppose precise values for the constants and variables used in the equations of motion. This, for example, is consistent with the description of ontic states having precise, definite values. Clark Glymour takes VD as a necessary criterion for determinism and cites Peirce and Reichenbach as examples of philosophers who have included this criterion in their analyses of determinism (1971, pp. 744-5). Since CPM is often taken as the paradigm example of a deterministic theory, it is natural that VD would come to be seen as part of the Laplacean vision for classical physics. It is only with the advent of quantum mechanics that questions were raised about the applicability of value determinateness to all of physics. ${ }^{7}$

\footnotetext{
${ }^{6}$ One might wonder about the relationship between DD and UE. After all, ordinary differential equations have theorems guaranteeing the existence and uniqueness of their solutions so that UE looks like a redundant requirement in these cases. However, by either underdetermining or overdetermining the conditions for such equations, uniqueness and/or existence may be lost. More generally, DD allows a great deal of freedom in choosing algorithms, including algorithms lacking uniqueness properties. Hence, UE is far from a redundant condition.
}

\footnotetext{
${ }^{7}$ Historically a fourth property known as absolute predictability completed the picture of determinism as conceived by Laplace, but the relationship of predictability to determinism is more subtle than typically realized and the type of predictability implied by DD, UE and VD is also much weaker than often conceived (Bishop
} 
The Laplacian vision certainly has the flavor of CPM, but as I have described it so far, it lacks the same precision. By looking at Bas van Fraassen's analysis of determinism (1989 and 1991), we can add precision to the vision as it comes to expression in CPM, connect it with the mathematical tools of physical theories, further motivate this formulation of determinism, and highlight once again the crucial role UE plays in the definition of determinism.

Van Fraassen begins his quantitative discussion by considering the following proposition (1989, p. 252):

(1) There is a function $\underline{\mathrm{f}}$ such that, for all times $\underline{\mathrm{t}}$ and positive numbers $\underline{\mathrm{b}}, \underline{\mathrm{s}}(\underline{\mathrm{t}}+\underline{\mathrm{b}})$ $=\underline{\mathrm{f}}(\underline{s}(\mathrm{t}), \underline{\mathrm{t}}, \underline{\mathrm{b}})$.

In (1), $\underline{\mathrm{s}}(\mathrm{t})$ is the state of a system at time $\underline{\mathrm{t}}$ and $\underline{\mathrm{f}}$ is a function that maps a state $\underline{\mathrm{s}}(\underline{\mathrm{t}})$ into a state that is $\underline{b}$ time units to the future. This represents a formalization of DD in terms of functions, where probability is explicitly excluded. As Russell pointed out, however, there is a problem with this type of functional definition (1953, pp. 401-2). At every instant in the history of the system, there exists an infinite number of functions describing the same time-evolution of the system in the past, but diverging in their descriptions in the future (after the time $\underline{t}+\underline{b}$, say). Hence, this definition of determinism is vacuous because there is never a case where there is a single unique function describing the evolution. ${ }^{8}$ Russell's suggestion for overcoming this difficulty is to characterize systems by a function making no explicit reference to time. His hope was that such a move would diminish, if not totally remove, the number of possible functions that agree with $\underline{\mathrm{f}}$ in their description of the time-evolution of the system up to the time t.

Apparently van Fraassen interprets Russell's suggestion as an attempt to define determinism in terms of actual trajectories alone (no possible trajectories allowed). He reconstructs Russell's suggestion as $(1989$, p. 252)

(2) There is a function $\underline{\mathrm{f}}$ such that for all times $\underline{\mathrm{t}}$ and numbers $\underline{\mathrm{b}}, \underline{\mathrm{s}}(\underline{\mathrm{t}}+\underline{\mathrm{b}})=\underline{\mathrm{f}}(\underline{\mathrm{s}}(\underline{\mathrm{t}})$, b).

On van Fraassen's analysis, for (2) to be an adequate definition of a deterministic model, it must represent a type of periodicity, but not the functional type where $\underline{s}(\underline{t})=\underline{s}(\underline{t}+\underline{b})$ say. Rather, the type of periodicity he has in mind is UE, where if a system returns to the same dynamical state at a different time, then it will repeat the same history of state transitions. In other words, (2) must be refined to

(2A) For any two distinct times $\underline{\mathrm{t}}, \underline{\mathrm{t}} \mathrm{N}$ if $\underline{\mathrm{s}}(\underline{\mathrm{t}})=\underline{\mathrm{s}}(\underline{\mathrm{t}} \mathrm{N})$, then $\underline{\mathrm{s}}(\underline{\mathrm{t}}+\underline{\mathrm{b}})=\underline{\mathrm{s}}(\underline{\mathrm{t}} \mathrm{N}+\underline{\mathrm{b}})$

if it is to be nonvacuous. Suppose our system $\underline{m}$ evolves in such a way that every state in the history of the trajectory is different. Then it would vacuously fulfill (2). An example would be an Epicurean atom always moving forward on a trajectory approximately along a line from the sun to the North

2003).

\footnotetext{
${ }^{8}$ Even if there is a single function describing the history of the system, such a function still does not imply the system is deterministic without the faithful model assumption.
} 
Star making an occasional indeterministic swerve. Without the periodicity requirement, (2) would pick out the Epicurean atom as deterministic which is clearly mistaken.

(2A) states explicitly that if a system returns to the same state at a different time, then it will follow the same history of state transitions and is an attempt to spell out the requirement of UE. Since (2A) implies the use of equations of motion as a prescription for the evolution of a system and satisfies VD in the context of CPM, we can understand the question van Fraassen wants to answer as being whether (2A) represents a sufficiently strong reading of UE to yield determinism. It turns out that (2A) is still not enough as there are formal counter examples of models with such periodic histories that are not deterministic (Montague 1974, pp. 337-9). Van Fraassen's conclusion is that we must take possible trajectories seriously when defining determinism-determinism is a modal notion (1989, p. 255).

What is needed is for any given state $\underline{\mathrm{s}}(\mathrm{t})$, all possible trajectories issuing forth from that state have the same history of state transitions. Van Fraassen writes this as (1989, p. 254)

(3) If $\underline{\mathrm{u}}$ and $\underline{\mathrm{v}}$ are possible histories, and $\underline{\mathrm{u}}(\mathrm{t})=\underline{\mathrm{v}}(\underline{\mathrm{t}} \mathrm{N}$ then for all positive numbers $\underline{\mathrm{b}}, \underline{\mathrm{u}}(\underline{\mathrm{t}}+\underline{\mathrm{b}})=\underline{\mathrm{v}}(\underline{\mathrm{t}} \mathrm{N}+\underline{\mathrm{b}})$.

How is this condition implemented? In CPM it is implemented by using a group of operators defined by the operation $\underline{\mathrm{U}}_{\mathrm{b}} \underline{\mathrm{u}}(\underline{\mathrm{t}})=\underline{\mathrm{u}}(\underline{\mathrm{t}}+\underline{\mathrm{b}})$, where there exists an inverse operator $\underline{\mathrm{U}}_{\mathrm{b}}{ }^{1}=\underline{\mathrm{U}}_{\mathrm{l}} \underline{\mathrm{b}}$ for $\mathrm{a}$ one-parameter family, and $\underline{\mathrm{U}}_{\underline{\mathrm{b}}} \underline{\mathrm{u}} \underline{\mathrm{u}}(\underline{\mathrm{t}}+\underline{\mathrm{b}})=\underline{\mathrm{u}}(\mathrm{t})$. This type of evolution operator corresponds to the three elements of the Laplacean vision described above. First, as the source for the equations of motion of CPM, it is a prescription for how to go from one state of the system to another (DD). Second, the state space trajectory governed by these operators is unique given any choice of initial state in the trajectory's history (UE). Third, the group operators and the resulting equations of motion possess the property of value determinateness as exhibited by the uniqueness and existence theorems for the differential equations of CPM (VD). Hence, group operators yield a precise nonvacuous realization of the Laplacean vision with UE playing a central role. By similar reasoning, we can see that semigroup operators can be used to achieve a unidirectional version of Laplacean determinism. ${ }^{9}$

\section{Determinism in Layers?}

In Stone's analysis of Laplacean determinism, DD, UE and VD are taken to be jointly necessary and sufficient conditions for a CPM model (or system) to be deterministic. This is intuitively easy to see: given a set of evolution equations and ICs and BCs, the state space trajectories of the model are uniquely determined by the evolution group $\underline{\mathrm{U}}_{\mathrm{b}}$. For CPM, DD, UE and VD and look like a packaged deal, given the nature of the states involved (point-like), the evolution equations and the uniqueness and existence theorems tying the two together.

In contrast, Kellert attempts to argue that DD, UE and VD are separable, representing layers of determinism (1993, pp. 50-62). Each property represents a layer of determinism, like the layers of an onion, and as we peel back the layers, we supposedly get closer and closer to the core of determinism. This analysis of determinism, however, raises questions about logical relations among states and the evolution equations in deterministic models as well as misidentifies the core

\footnotetext{
${ }^{9}$ Semigroups share all the properties of groups except that they lack an inverse.
} 
property of determinism as we will see.

Kellert treats VD as the outermost layer of determinism. Suppose we drop this condition. If one thinks that determinateness and determinism are closely related-as Glymour (1971) suggests-then dropping VD would automatically guarantee indeterminism in a physical description. Although VD appears to apply to CPM, one might worry that quantum mechanics implies that physical variables do not have sharp or definite values. However, it is possible to revise determinism to allow for set- and interval-valued properties evolving along uniquely determined paths (Fine 1971; Teller 1979; Earman 1986, 217-8). Properties DD and UE can be applied to the evolution of interval-valued quantities. So DD, UE and VD, then, do not form a set of jointly necessary and sufficient conditions for determinism.

Peeling away VD, then, does not appear to be sufficient to render a model indeterministic and it looks like Kellert's analysis of determinism as a layered concept proceeds straightforwardly in this respect. However there is a subtlety involved here. Recall that states are typically taken to be values of the key variables characterizing the system at some time $t$ and are represented as points in state space. So a given state $\underline{\mathrm{s}}\left(\mathrm{t}_{a}\right)$ characterizes a system at the particular time $\underline{\mathrm{t}}_{\mathrm{a}}$. But when we consider states in conjunction with the evolution equations governing the history of state transitions, there is a very important dependence ignored in Kellert's analysis. The three properties DD, UE and VD are derived from CPM, where states are conceived as points in states space. By dropping $\mathrm{VD}$, we have actually introduced a change in the notion of state from that of point-valued to that of interval-valued. For states characterized by interval-valued variables, the state transitions governed by the evolution operators would no longer be point-valued trajectories in state space, but, rather, "tubes" connecting an initial interval-valued state with some later interval-valued state in some appropriate state space. Given the same initial state, DD and UE would still imply that such tubes exhibit a unique history of state transitions ${ }^{10}$, so determinism still holds, but point-valued states are no longer elements of the models. So there is a logical connection between the properties DD, UE, and VD, on the one hand, and the notion of state on the other. Particular equations of motion (DD) presuppose particular specifications of the states they govern (e.g., point-like as in VD) in order for the models to represent well-posed mathematical problems guaranteeing the existence of unique solutions (UE). The definition of states, then, in a physical theory are not arbitrary, but tied to the evolution equations governing the states. Making a change in the nature of the state entails a corresponding change in the nature of the evolution equations and modifying the evolution equations generally necessitates a corresponding redefinition of the notion of state.

The second layer in Kellert's analysis of determinism is UE. He argues that UE can be separated from determinism because chaotic systems can amplify quantum fluctuations due to sensitive dependence leading to violations of UE (1993, pp. 69-75; see also Hobbs 1991, p. 157). Briefly, the reasoning runs as follows. Given two chaotic systems of CPM in nearly identical initial states (e.g., specification of the initial positions and velocities), they will evolve in radically different ways in a relatively short time period as the slight differences in initial conditions are amplified (socalled sensitive dependence). There is no known lower limit to this sensitivity, thus nothing to prevent the possibility of chaotic macroscopic systems being sensitive to quantum fluctuations. If chaotic macroscopic systems are sensitive to quantum fluctuations, then such fluctuations would generate slight differences in initial conditions for such systems yielding different histories of state

\footnotetext{
${ }^{10}$ Recall that probabilities are explicitly disallowed under DD.
} 
transitions for chaotic macroscopic systems (Bishop and Kronz 1999). Hence UE must fail for chaotic models in CPM.

The question of whether such sensitivity arguments do not run afoul of DD's restriction on no probabilities is a delicate matter. However, these kinds of sensitivity arguments depend crucially on how quantum mechanics itself as well as measurements are interpreted (Bishop and Kronz 1999). Furthermore, although in the abstract sensitivity arguments do correctly lead to the conclusion that the smallest of effects can be amplified, applying such arguments to concrete physical systems shows that the amplification process may be severely constrained. For example investigating the role of quantum effects in the process of friction in sliding surfaces indicates quantum effects might be amplified by chaos to produce a difference in macroscopic behavior only if the fluctuations are large enough to break molecular bonds and are amplified quickly enough (Bishop forthcoming).

These questions aside, what would it mean for the concept of determinism if UE were dropped? I offered reasons in section 3 as to why UE is crucial for any conception of determinism. The crucial question, then, is whether by dropping UE determinism would be lost if all we now have is the concept of an evolution equation (i.e., only DD remains). To see this, we can build what van Fraassen calls a set of "indeterministic counterparts" to the group operators mentioned above that explicitly lack UE (van Fraassen 1991, p. 51). Let $\underline{S}$ be a subset of the state space and $\underline{b}$ a positive number. Then

$\underline{\mathrm{T}}_{\mathrm{b}-}(\underline{\mathrm{S}})=\left\{\underline{\mathrm{x}}\right.$ : for some possible ${ }^{*}$ trajectory $\underline{\mathrm{u}}$, time $\underline{\mathrm{t}}$, and state $\mathrm{y}$ in $\left.\underline{\mathrm{S}}, \underline{\mathrm{u}(\mathrm{t})}\right)=\underline{\mathrm{y}}$ and $\mathrm{u}(\underline{\mathrm{t}}+\underline{\mathrm{b}})=$ $\underline{\mathrm{x}}\}$;

$\underline{\mathrm{T}}_{\mathrm{b}-}(\underline{\mathrm{S}})=\{\underline{\mathrm{y}}$ : for some possible* trajectory $\underline{\mathrm{u}}$, time $\underline{\mathrm{t}}$, and state $\underline{\mathrm{x}}$ in $\underline{\mathrm{S}}, \underline{\mathrm{u}(\mathrm{t})})=\underline{\mathrm{y}}$ and $\mathrm{u}(\underline{\mathrm{t}}+\underline{\mathrm{b}})=$ $\underline{\mathrm{x}}$,

where a trajectory $\underline{\mathrm{u}}(\mathrm{t})$ is possible* relative to $\underline{\mathrm{v}}(\underline{\mathrm{t}})$ exactly when $\underline{\mathrm{u}}(\underline{\mathrm{t}})=\underline{\mathrm{v}}(\underline{\mathrm{t}})$ for all $\underline{\mathrm{t}} \# \underline{\mathrm{t}}_{1}$, but may disagree for all $\underline{t}>\underline{t}_{1}$. The operation $\underline{T}_{b} \underline{T}_{b-}(\underline{S})$ does not return the original state in $\underline{S} .{ }^{11}$ Furthermore these operators require a change in the notion of possibility from the standard one used in groups and semigroups. The operators $\underline{T}$ yield a structure on state space defining a set of possible trajectories that can act as continuations of $\underline{\underline{u}}$ beyond time $\underline{\mathrm{t}}_{1}$. Any one of the candidates is a possible continuant and no one is guaranteed to be the continuant.

Thus UE is lost with no explicit introduction of probability into any equations, hence, there is no immediate revision to DD which explicitly rules probabilities out. However, clearly if UE is dropped, then the algorithm in DD is either vacuous (recall (2) and (2A) above) or one-to-many: given the same state $\underline{\mathrm{s}}(\underline{\mathrm{t}})$ for any $\underline{\mathrm{t}}<\underline{\mathrm{t}}_{1}$, the algorithm can map into any possible ${ }^{*}$ state $\underline{\mathrm{s}}(\underline{\mathrm{t}} \mathbb{N})$ for $\underline{\mathrm{t}} \mathrm{N} \vee$ $\mathrm{t}_{1}$. If the key intuition is that deterministic evolution must eschew probabilities-as seems to be Kellert's view (1993, p. 75)-then that intuition is certainly preserved by dropping UE and keeping DD. However, the remaining conception of determinism turns out to be simply a methodological injunction to use differential equations, say, without any inherent stochastic elements, an approach that has its own problems (cf. Bishop 2002, pp. 26-28). Even if DD remains unmodified, we have peeled the crucial layer of determinism away by dropping UE (compare Earman 1986, pp. 12-13). As illustrated and argued above in section 3, UE is the core of determinism, representing an anvil on

\footnotetext{
${ }^{11}$ They form a semigroup of operators (see Bishop 2002, pp. 13-14)
} 
which we fashion our physical theories and models.

Suppose, in contrast to Kellert, we drop DD's restriction on probabilities, while maintaining $\mathrm{UE}$ as the core of determinism (our anvil). Determinism as UE is still preserved since it is still possible to have evolution equations that govern the transitions of probabilities such that starting with the same initial probability distribution (initial state), we have the same history of transitions to a unique final probability distribution (final state). An example of such a situation would be statistical mechanics. But notice again that this would involve a further revision to the nature of the states composing the state space: the states are now probability distributions, rather than point-valued or interval-valued quantities. So UE holds whether or not we have evolutions equations for pointvalued states or for probability distributions. The different kinds of evolutions equations represent the various kinds of theories or models we can fashion on the anvil of UE, ensuring that such theories and models are deterministic.

The interplay between the notion of state and the types of equations in DD also helps to alleviate a worry for UE raised by Robert Batterman (1993). He notes that UE is "generally false for so-called time-dependent Hamiltonian systems" (1993, p. 50). I take it that the worry here is with behaviors such as hysteresis effects, where physical variables become multivalued. Again, what is important to note here is that the notion of state corresponding to these equations. In this case the state is such that it never takes on multiple values of a variable (the velocity, say) at the same time t. The notion of state is, perhaps, more sophisticated than in Newtonian dynamics, but there is nothing in principle that prevents enriched notions of state and state space, along with the corresponding evolution equations, from fulfilling UE in these cases.

As a final set of remarks, the connection between the properties of determinism and the notion of state often appears to be neglected. A recent example is David Albert's (2000) discussion regarding determinism and time-reversal invariance. Albert (2000, pp. 9-11) advocates a view that states should be considered logically, conceptually, and metaphysically independent. This is to say that the information encoded in the state $\underline{\mathrm{s}}(\underline{\mathrm{t}})$ does not determine or imply anything about any other states at any other times if we consider states in and of themselves. If one considers Newtonian particle mechanics, specifying just instantaneous particle positions fulfills this criterion fine. If, in addition, one specifies instantaneous particle momenta as well, then this independence is violated, because the definition of instantaneous velocity involves infinitesimal differences in position at different times, since momentum in Newtonian mechanics is defined as velocity-the derivative of position with respect to time-multiplied by mass. As John Earman has pointed out, physicists usually do not require this kind of strict independence. Defining states in CPM as $\underline{\mathrm{s}}(\underline{\mathrm{x}}(\underline{\mathrm{t}})$, $\underline{\operatorname{mv}}(\underline{\mathrm{t}}))$, where $\underline{\mathrm{x}}$ is particle position and $\underline{\mathrm{mv}}$ is particle momentum, such a state is not independent of all the other states in some specified time interval, namely it fails in just the way needed to define $\underline{\mathbf{v}}$ as an instantaneous velocity (Earman 2002, p. 246). Still, merely specifying the state $\underline{\mathrm{s}}\left(\underline{\mathrm{x}}\left(\underline{\mathrm{t}}_{a}\right), \underline{\mathrm{mv}}\left(\underline{t}_{a}\right)\right)$ does not determine the values of all other states in state space for all times, but only those in a small neighborhood about s. On the other hand, if one considers the Hamiltonian formulation of CPM, where the generalized momentum is defined as a derivative of the system's Lagrangian (roughly a function specifying the total energy of the system), then the generalized momentum need not be of the same form as Newtonian momenta, so one may be able to specify both the generalized coordinates and the generalized momenta such that states maintain the conceptual independence Albert intends.

It is also logically possible that a system might have the same state in two different worlds, 
but not have the same history of state transitions. This, perhaps, is the notion of logical independence that Albert has in mind. However, in physically possible worlds where the laws are deterministic, the states would not be logically independent. In particular, under the Laplacean vision of determinism for CPM, the states are not logically independent of the laws governing their history of transitions (DD), and, consequently, they are not strictly logically independent of each other, being linked via the relevant evolution equations, whether the Newtonian or the Lagrangian formulation is used. The specification of one of these states plus the dynamical equations of motion would fix the entire history of state transitions so long as the definition of state includes the instantaneous position and momentum. So the fact that particular forms of determinism presuppose particular notions of state undermines the kind of strong form of independence Albert advocates in contrast to the typical view of physicists.

The importance of this interdependency of states and equations of motion for determinism can be seen in an example given by van Fraassen (1989, p. 256). Consider a pendulum whose motion is constrained to a plane and whose states $\underline{s}$ are only characterized by bob position in order to enforce strict independence of states. Suppose that the bob has a particular location at some time, whose coordinates are $(1,1)$, say. Since the pendulum is a periodic system, we know it will return to this location again and again in regular fashion. Characterizing bob positions as a function of (discrete) time $\underline{\mathrm{s}}(\mathrm{t})$, then $\underline{\mathrm{s}}(10)=(1,1)$. Suppose that $\underline{\mathrm{s}}(20)=(1,1)$. Does it follow from this that $\underline{\mathrm{s}}(11)=\underline{\mathrm{s}}(21)$ ? No, because whether the bob is traveling in the same direction at these two times is indeterminate. Given a time sequence of positions $\underline{\mathrm{s}}(10), \underline{\mathrm{s}}(20), \underline{\mathrm{s}}(30), \ldots$ there is no way of determining the values of the other positions. The pendulum, then, is periodic, but not deterministic if the state space is simply configuration space $(\underline{\mathrm{x}}(\mathrm{t}))$. Of course, when we examine the pendulum's equation of motion, we see that it presupposes states characterized by $(\underline{\mathrm{x}}(\underline{\mathrm{t}}), \underline{\mathrm{mv}}(\underline{\mathrm{t}}))$. So the state space that allows full specification of the state of the pendulum bob is phase space, requiring both position and momentum, and determinism is restored.

In contrast to the view presented here, Albert advocates viewing determinism in CPM as a "connection between all the states of the world at all times and all the states of the world throughout any arbitrarily small time-interval" (Albert 2000, p. 11). This means that one must specify the states $\underline{\mathrm{s}}\left(\underline{\mathrm{x}}\left(\underline{\mathrm{t}}_{\mathrm{a}}\right)\right)$ at some time $\underline{\mathrm{t}}_{\mathrm{s}} \underline{\text { and }}$ the "rates at which those positions are changing [momentum] in the immediate vicinity of that time" in order to have a deterministic connection with the positions of the particles at any other time" (Albert 2000, p. 11). In this way one could overcome the problem of determinism in the previous pendulum example, because the equation of motion requires a specification of both an initial position and a rate of change of that position at some time $\underline{t}_{s}$ in order for determinism to hold for the system. So, on Albert's view, since the CPM equations of motion require both initial position and velocity information, and since the $\underline{\mathrm{s}}(\underline{\mathrm{x}}(\underline{\mathrm{t}}-\mathbf{a}))$ contains no velocity information, one must also specify how $\underline{\mathrm{s}}\left(\underline{\mathrm{x}}\left(\underline{\mathrm{t}}_{-}\right)\right)$is changing in a small neighborhood in time about $\underline{\mathrm{t}}_{\mathrm{s}}$.

As noted above, however, states are not strictly logically independent of each other, being related to one another via the equations of motion, so the drive to formulate CPM in such a way that particle states have the strict independence Albert wants lacks motivation. It is also the case that on Albert's proposal, given a state $\underline{\mathrm{s}}\left(\underline{\mathrm{x}}\left(\mathrm{t}_{a}\right)\right)$ one could not say whether it represented a particle state in motion or at rest, whereas on the usual physicist's account this can be read off the state. With respect to determinism, however, the greatest difficulty with Albert's proposal is that if the state is restricted to $\underline{\mathrm{s}}\left(\underline{\mathrm{x}}\left(\underline{\mathrm{t}}_{-}\right)\right)$and only $\underline{\mathrm{s}}\left(\underline{\mathrm{t}}_{0}\right)$ is specified along with the equations of motion, then the system is not deterministic (as in the example above). Under this proposal, determinism does not 
have the properties DD, UE and VD falling out of the kinds of laws we take to govern CPM. Rather, one must decide what additional information is needed beyond $\underline{\mathrm{s}}\left(\mathrm{t}_{0}\right)$ in order to restore determinism for CPM, but this renders the specification of determinism ad hoc, whereas the usual physicist's way of specifying states leaves the question of determinism internal to the theory or model in question and well-motivated.

\section{Concluding Comments}

From this brief exploration of Laplacean determinism, we can see that the notion of determinism as a layered concept is more problematic than first meets the eye. DD, UE and VD might be jointly necessary and sufficient conditions for models of CPM to be deterministic. To drop either DD or VD might be appropriate for other kinds of models-so these three conditions cannot be necessary for Laplacean determinism simpliciter-but this also implies that the underlying notion of state changes in a corresponding way. For example, dropping VD leads to a change in state from pointvalued to interval valued. This might be appropriate for particular kinds of meteorological models, where variables like temperature and pressure for atmospheric states are specified over some interval due to measurement limitations and these interval-valued states are then evolved forward in time. While still being deterministic, this epistemic description could be related to an underlying ontic description in terms of microscopic point-valued states (Atmanspacher and Kronz 1999). ${ }^{12}$ As another example, in statistical mechanics, dropping DD might mean probability densities are being used in an appropriate coarse-grained macroscopic description of the system tied to the precision of our observations. While still being deterministic, this epistemic description could be related to an underlying ontic description in terms of microscopic point-valued states. Alternatively, there might be cases where an ontic description in terms of probabilities is appropriate when underlying pointvalued states are undefinable. Hence, we have a different conception of determinism suited for modeling physical systems other than those of CPM.

In contrast, dropping UE does not appear to imply a corresponding change to the notion of state. For example, whether one uses point-valued or interval-valued quantities-corresponding to maintaining or dropping VD-specifying the same initial state does not guarantee evolution to a unique final state because the mapping between states is now one-to-many. Determinism is no longer valid for such models. Speaking picturesquely, UE represents an anvil and different combinations/modifications of DD and VD represent different properties relevant for different models we might fashion on this anvil.

These observations suggest that a layered conception of determinism is inappropriate. Rather, we should think in terms of different deterministic models applicable to different kinds of systems all sharing the core property UE. Further, the notion of state is more closely tied to the kind of system being investigated than is usually considered in discussions of determinism. So when investigating determinism by adding or dropping such properties as DD and VD, corresponding changes to the appropriate notion of state-and, perhaps, the state space itself-also need to be considered.

\section{Acknowledgments}

\footnotetext{
${ }^{12}$ It is possible to have probabilistic descriptions that are ontic with respect to other descriptions (Atmanspacher and Kronz 1999; Atmanspacher, Bishop and Amann 2002, pp. 64-67).
} 
Part of this essay was presented at a seminar in the Institute for the History and Foundations of Mathematics and the Natural Sciences at the University of Utrecht, providing much clarification and insight. Particular thanks is owed Iain Martel, Jos Uffink and Veiko Plage for helpful questions and clarifications. Support from the Alexander von Humboldt Foundation as well as the Federal Ministry of Education and Research and the Program for the Investment in the Future of the German Government are gratefully acknowledged.

\section{References}

Albert, D. (2000), Time and Chance. Cambridge, MA: Harvard University Press. Atmanspacher, H. (1994), "Is the Ontic/Epistemic Distinction Sufficient to Describe Quantum Systems Exhaustively?" in K. Laurikainen, C. Montonen and K. Sunnarborg (eds.), Symposium on the Foundations of Modern Physics 1994. Gif-surYvette: Editions Frontières, pp. 15-32.

Atmanspacher, H., Bishop, R. C. and Amann, A. (2002), "Extrinsic and Intrinsic Irreversibility in Probabilistic Dynamical Laws," A. Khrennikov (ed.), Quantum Probability and White Noise Analysis Volume XIII. Singapore: World Scientific, 2002, pp. 50-70.

Atmanspacher, H., and Kronz, F. K. (1999), "Relative Onticity,"' in H. Atmanspacher, A. Amann and U. Müller-Herold (eds.), On Quanta, Mind and Matter: Hans Primas in Context. Dordrecht: Kluwer Academic Publishers, pp. 273-294.

Benacerraf, P. (1962), “Tasks, Supertasks, and Modern Eleatics,” Journal of Philosophy LIX: 765-784.

Batterman, R. W. (1993), "Defining Chaos," Philosophy of Science 60: 43-66.

Bishop, R. C. (2002), "Deterministic and Indeterministic Descriptions," in A. Atmanspacher and R. C. Bishop (eds.), Between Chance and Choice. Thorverton: Imprint Academic, pp. 5-31.

Bishop, R. C. (2003), “On Separating Predictability and Determinism,” Erkenntnis 58: 169188.

Bishop, R. C. (forthcoming), "What Could Be Worse than the Butterfly Effect?"

Bishop, R. C. and Kronz, F. K. (1999), "Is Chaos Indeterministic?” in M. Dalla Chiara, R. Guintini and F. Laudisa (eds.) Language, Quantum, Music: Selected Contributed Papers of the Tenth International Congress of Logic, Methodology \& Philosophy of Science, Florence, August 1995. London: Kluwer Academic Publishers, 129-41.

d'Espagnat, B. (1994), Veiled Reality: An Analysis of Present-Day Quantum Mechanical Concepts. Reading, MA: Addison-Wesley.

Earman, J. (1986), A Primer on Determinism. Dordrecht, The Netherlands: D. Reidel Publishing.

Earman, J. (2002), “What Time Reversal Invariance Is and Why It Matters,” International Studies in the Philosophy of Science 16: 245-264.

Earman, J. and Norton, J. (1996), "Infinite Pains: The Trouble with Supertasks," in A. Morton and S. Stich (eds.), Benacerraf and His Critics. Oxford: Blackwell Publishers, pp. 231-261.

Fine, A. (1971), "Probability in Quantum Mechanics and Other Statistical Theories," in M. Bunge (ed.) Problems in the Foundations of Physics. New York: Springer-Verlag. 
Glymour, C. (1971), 'Determinism, Ignorance, and Quantum Mechanics', The Journal of Philosophy 68: 744-751.

Hobbs, J. (1991), "Chaos and Indeterminism," Canadian Journal of Philosophy 21:141-164.

Kellert, S. (1993), In the Wake of Chaos. Chicago: University of Chicago Press.

Laplace, P. (1814/1951), A Philosophical Essay on Probabilities. New York: Dover

Publications.

Montague, R. (1974), Formal Philosophy: Selected Papers of Richard Montague, R. H.

Thomason (ed.). New Haven: Yale University Press.

Primas, H. (1990), "Mathematical and Philosophical Questions in the Theory of Open and Macroscopic Quantum Systems," in A. Miller (ed.), Sixty-Two Years of Uncertainty. New York: Plenum, pp. 233-57.

(1994), "Endo- and Exotheories of Matter," in H. Atmanspacher and G. Dalenroot (eds.) Inside Versus Outside. Berlin: Springer-Verlag, pp. 163-93.

Russell, B. (1953), "On the Notion of Cause, with Applications to the Free-Will Problem" in H. Feigl and M. Brodbeck (eds.), Readings in the Philosophy of Science. New York: Appleton-Century-Crofts Inc, pp. 387-418.

Scheibe, E. (1964/1973), The Logical Analysis of Quantum Mechanics. Oxford: Pergamon Press.Stone, M. A. (1989), "Chaos, Prediction and Laplacean Determinism," American Philosophical Quarterly 26: 123-31.

Teller, P. (1979), "Quantum Mechanics and the Nature of Continuous Physical Magnitudes," Journal of Philosophy 76:345-61.

Thomson, J. (1954-55), "Tasks and Supertasks," Analysis XV: 1-13. van Fraassen, B. (1989), Laws and Symmetry. Oxford: Clarendon Press. (1991), Quantum Mechanics: An Empiricists View. Oxford: Clarendon

Press. 